Georgetown University Institutional Repository http://www.library.georgetown.edu/digitalgeorgetown

The author made this article openly available online. Please tell us how this access affects you. Your story matters.

Tanner, R. J. \& Carlson, K. A. (2009). Unrealistically Optimistic Consumers: A Selective Hypothesis Testing Account for Optimism in Predictions of Future Behavior. Journal of Consumer Research, 35(5), 810-822.

doi: $\underline{10.1086 / 593690}$

Collection Permanent Link: hdl.handle.net/10822/707923

(C) 2009 University of Chicago Press.

This material is made available online with the permission of the author, and in accordance with publisher policies. No further reproduction or distribution of this copy is permitted by electronic transmission or any other means. 


\title{
Unrealistically Optimistic Consumers: A Selective Hypothesis Testing Account for Optimism in Predictions of Future Behavior
}

\author{
ROBIN J. TANNER \\ KURT A. CARLSON*
}

\begin{abstract}
We propose that when predicting future behavior, consumers selectively (but unwittingly) test the hypothesis that they will behave ideally. This selective hypothesis testing perspective on unrealistic optimism suggests that estimates of future behavior should be similar to those made by individuals who assume that conditions will be ideal. Moreover, consumers who initially provide estimates assuming that conditions will be ideal should recognize that the world is not ideal and so should test a more realistic hypothesis. In line with these predictions, we find that idealworld estimates (e.g., In an ideal world, how often will you exercise next week?) do not differ from standard estimates (e.g., How often will you exercise next week?). We also find that individuals who initially estimate their behavior in an ideal world subsequently make more realistic predictions.
\end{abstract}

\begin{abstract}
A wealth of research has found that people view the future through rose-tinted glasses. In general, people tend to believe that they are more likely than others to undertake positive behaviors or to experience positive events and believe that they are less likely to undertake negative behaviors or to experience negative events (Armor and Taylor 2002; Burger and Burns 1988; Taylor and Brown 1988; Weinstein 1980). Such unrealistic optimism occurs whether people are making comparative judgments (e.g., "Compared to an average student, how likely are you to catch an STD in the next year?") or absolute judgments (e.g., "How likely are you to catch an STD in the next year?"). Typical examples include optimistic estimates of whether one will get a good first job (Weinstein 1980), avoid an unwanted pregnancy (Burger and Burns 1988), contract lung cancer (Perlof and Fetzer 1986), or be in a car accident (McKenna 1993).
\end{abstract}

Robin Tanner is assistant professor of marketing at the University of Wisconsin, Madison, WI 53706 (rtanner@bus.wisc.edu). Kurt Carlson is assistant professor of marketing at Duke University, Durham, NC 27708 (kurt.carlson@duke.edu). E-mail correspondence can be sent to either author. The authors acknowledge the helpful input of the editor, associate editor, and reviewers. They would like to thank Jim Bettman, Tanya Chartrand, Rick Larrick, John Lynch, Jay Russo, and Larry Sanna for valuable feedback on earlier drafts of this article. The authors contributed equally to this article. This article was partially based on the first author's dissertation at Duke University.

John Deighton served as editor and Ann McGill served as associate editor for this article.

Electronically published October 8, 2008
Interestingly, unrealistic optimism has received little attention from consumer researchers. This is surprising since the potential implications of unrealistic optimism for consumer decision making are considerable. For example, if consumers' beliefs about their own future behavior are unrealistically optimistic and if consumers use these beliefs as inputs to product valuation, then they may unwittingly overestimate or underestimate how much they will actually value a product in the future. Put differently, to accurately value a product or service that will be consumed at a future date, consumers must accurately predict their behavior at that future date.

This article focuses on consumers' predictions of their future behavior, which we find are both unrealistically optimistic and influential on downstream decisions. We trace the origin of these unrealistically optimistic expectations to a process of selective hypothesis testing, wherein consumers initially adopt a tentative hypothesis of idealistic future behavior and then selectively recruit information to support it. From this view, we predict that estimates of a specific future behavior should be nearly as optimistic as estimates of the same behavior made assuming ideal conditions. Moreover, it should be possible to get consumers to abandon their tentative hypothesis by making the contrast between ideal and real salient. That is, consumers who first estimate their behavior in an ideal world (i.e., test a hypothesis of ideal behavior) should realize for themselves that the world is not ideal and so should test a more realistic hypothesis when estimating their actual behavior. Consequently, these individuals should provide more realistic estimates of actual 
behavior than individuals who simply estimate their behavior (i.e., without initially estimating it in an ideal world).

The remainder of this article is as follows. In the next section, we briefly review the literature on unrealistic optimism and introduce our selective hypothesis testing view. Following this, we present four studies that explore predictions derived from the selective hypothesis testing perspective. We conclude with discussions of theoretical and practical implications.

\section{BACKGROUND}

The robustness of unrealistic optimism has spawned numerous explanations, which can be broadly categorized as either motivational or nonmotivational. Motivational accounts contend that unrealistic optimism serves a desire to protect and bolster the self (Hoorens 1995; Taylor and Armor 1996). For example, Wills (1981) suggests that individuals selectively compare themselves with targets that produce esteem-enhancing estimates (e.g., a comparison to an individual who has a higher risk of contracting an STD) and thus support their desired conclusions (e.g., that one is not at risk). In contrast, nonmotivated accounts, most of which invoke egocentrism, generally contend that cognitive processes are sufficient to produce unrealistically optimistic estimates. For example, Weinstein (1987) suggests that individuals arrive at unrealistically optimistic self-estimates because they focus on the causal actions they will take in future scenarios while failing to recognize similar actions that others might take. Similarly, Kahneman and Tversky (1979) argue for a self-centric mechanism when they suggest that the planning fallacy is caused by individuals taking an "inside" perspective, focusing on positive behaviors, while failing to consider less optimal behaviors. They conclude that mental simulations about future scenarios appear to be biased toward the positive, with an "idealized" version of the future often emerging (Kahneman and Tversky 1982).

Building on the above, we propose a view that borrows from both motivational and cognitive accounts. Specifically, we propose that individuals faced with estimating their future behavior are motivated to adopt a hypothesis of ideal behavior and then selectively recruit information that accords with this hypothesis. Below we describe this account in detail.

\section{A Selective Hypothesis Testing Account of Unrealistic Optimism}

The estimation of one's future behavior involves the assembly and integration of various elements of information about one's past behaviors, one's expected future state of mind and body, and the likely state of the world. Theories of how this assembly and integration occur vary from data driven (i.e., bottom-up theories) to hypothesis driven (i.e., top-down theories). In reality, the process is almost certainly a mixture of the two, with salient data giving rise to a working hypothesis that guides subsequent information acquisition and integration. For example, when one is esti- mating one's future behavior, the working hypothesis might take the form of a desirable outcome of one's future behavior. The top-down influence of such a working hypothesis greatly simplifies the task of producing an estimate. That is, by focusing on the working hypothesis and those similar to it, the judge can greatly reduce the set of information that is perceived as relevant for the estimation at hand. Individuals who simplify processing in this way are said to be engaging in selective hypothesis testing (for a review see Sanbonmatsu et al. [1998]).

In line with the above, it has long been argued that consumer beliefs are often constructed around tentative hypotheses (Hoch and Deighton 1989). Thus, consumers faced with predicting their future behavior are likely to do so by constructing estimates around tentative hypotheses of their future behavior. Because there are many possibilities for one's future behavior, consumers often simplify matters and focus on a single tentative hypothesis. We propose that the desire to think positively about oneself, which dominates motivational theories of unrealistic optimism, causes consumers to adopt a hypothesis of ideal behavior. That is, consumers adopt the hypothesis that they will behave as they would if there were no substantial obstacles preventing them from behaving as they wish to. For example, if asked to predict whether he will donate blood at an upcoming drive, an individual might adopt a tentative hypothesis that he will indeed have the time and motivation needed to do so. Recent research supports this contention. Newby-Clark (2005) had participants provide best, worst, and realistic scenarios as to how they would change their exercise behavior over the next month and found that the realistic estimates most resembled best-case estimates. In a similar vein, Williams and Gilovich (2006) found that estimates of typical performances were similar to estimates of best performance.

Building from theories of selective hypothesis testing (Gettys and Fisher 1979; Sanbonmatsu et al. 1998), we posit that consumers selectively test their (working) ideal hypothesis by focusing on evidence that supports it and neglecting evidence that contradicts it. For example, in the blood donation example, individuals would gather information that supports their donating blood (such as past memories of donation or anticipation of the satisfaction that donation will bring) and neglect information that is discordant with the hypothesis (such as their tendency to procrastinate or thoughts about the pain of the needle). Indeed, research suggests that consumers access information from memory in a way that is consistent with selective hypothesis testing (Deighton 1984; Posavac et al. 2004). The ultimate consequence of this proposed process is that predictions of future behavior will be unrealistically optimistic (e.g., a likelihood of donating blood that is too high).

Though selective hypothesis testing has been studied extensively since Wason's (1971) initial demonstrations, very little is known about how to reduce it in situations in which judgments are made quickly. That is, while there is an emerging consensus that selective hypothesis testing can be 
exacerbated by adding time pressure or increasing cognitive load (Cronley et al. 2005; Smith et al. 2007), we know of no empirical evidence indicating that selective hypothesis testing can be turned off. Since one of our primary objectives is to explore whether consumers can be led to make more realistic predictions of future behavior, we required a different approach. Specifically, we examine whether we can get individuals to think more realistically about future behavior not by turning off selective hypothesis testing, but instead by changing the hypothesis they are testing. To this end, we developed the two-condition design below.

\section{Research Overview}

Participants in the standard request condition answer a focal question of interest pertaining to an absolute estimate of a future behavior (e.g., "How many times will you exercise next month?"). Before answering this focal question, participants in the ideal-first condition provide an idealistic estimate (e.g., "In an ideal world, how many times would you exercise next month?"). This "ideal-world" question serves two purposes. First, since responses to it are by definition idealistic, we can use them to determine if standard condition responses are idealistic. That is, if optimistic predictions of future behavior stem from selective testing of an idealistic hypothesis, then estimates made by individuals who assumed that things would be ideal should be similar to standard condition estimates. Second, answering the ideal-world question should make salient that the real world is not ideal. This should cause individuals in the ideal-first condition to adopt and test a more realistic hypothesis when contemplating the focal question. As such, when compared to individuals who do not answer the ideal-world question, those who do should provide more realistic estimates of future behavior, report more realistic thoughts, and potentially make improved downstream judgments.

It is worth noting that getting individuals to be realistic is not as easy as it might seem. There is ample evidence that individuals are either unwilling or unable to let go of idealistic self-assessments. For example, Kruger and Gilovich $(2004,334)$ found that individuals reduced self-ratings by only a small amount (leaving them still unrealistically optimistic) when they were told to base their "ratings exclusively on overt behavior, observable actions, or tangible accomplishments" and to ignore "other components of the trait such as good intentions, plans for the future, or unfulfilled potential." Indeed, even when individuals can identify optimism in an estimate, they are unwilling to adjust for it (Soll, Larrick, and Zhu 2006).

In what follows, we examine these predictions for several behaviors. Study 1 combines three experiments that explore estimates of blood donation likelihood, exercise frequency, and savings discipline. Study 2 examines a downstream consequence of beliefs about future exercise behaviors, namely, willingness to pay for a treadmill. Study 3 concerns predictions of product usage and explores whether the effect of prior consideration of ideal behavior is moderated by expertise. Finally, study 4 explores the planning fallacy and whether more realistic predictions come at the cost of poorer performance.

\section{STUDY 1: PREDICTING CONSEQUENTIAL BEHAVIORS}

This first study explores the estimation of future behavior in the domains of blood donation, exercise frequency, and savings discipline. It consists of three experiments (and some replicates), each of which was run separately. However, they are reported together to avoid repetition in the description both of their common method and in the pattern of results.

\section{Method}

Participants were 76 undergraduates (in the blood donation experiment), 176 adult attendees at a dance performance (in the exercise experiment), and 35 undergraduates (in the savings experiment). The same basic design applied in each experiment. Participants were randomly assigned to either the standard request or the ideal-first condition and thus answered just the focal question or the ideal-world and focal questions, respectively. In each case, participants read a brief scenario introduction before proceeding to the questions of interest. The specific questions asked in each domain are shown in table 1. In addition to answering questions about their own behavior, those in the blood donation experiment estimated peer donation likelihood after stating their own intent. In the exercise experiment, a control group estimated how many times the typical dance performance attendee would exercise per week over the next month. These peer estimates give us a benchmark against which to assess optimism in self-estimates.

\section{Results}

The data from all three experiments (see table 2) reveal a consistent pattern. Specifically, there was no difference between focal question estimates in the standard request condition and ideal-world estimates. This is consistent with the idea that participants in the standard condition selectively tested a hypothesis of ideal behavior, resulting in idealized estimates. It was also the case that in both the exercise and blood donation experiments (where peer estimates were collected), focal estimates in the standard request condition were higher than estimates of peer performance. These data are indicative of unrealistic optimism.

Additionally, in every domain, focal question estimates by participants in the ideal-first condition were lower than focal question estimates in the standard condition, and they did not differ from peer estimates in the two domains in which peer estimates were collected (i.e., blood donation and exercise). In other words, those who initially estimated their ideal-world behavior gave responses to the focal question that were less optimistic and nearer to their estimates of peer performance than those who just answered the focal question. 
TABLE 1

BEHAVIOR PREDICTION QUESTIONS-STUDY 1

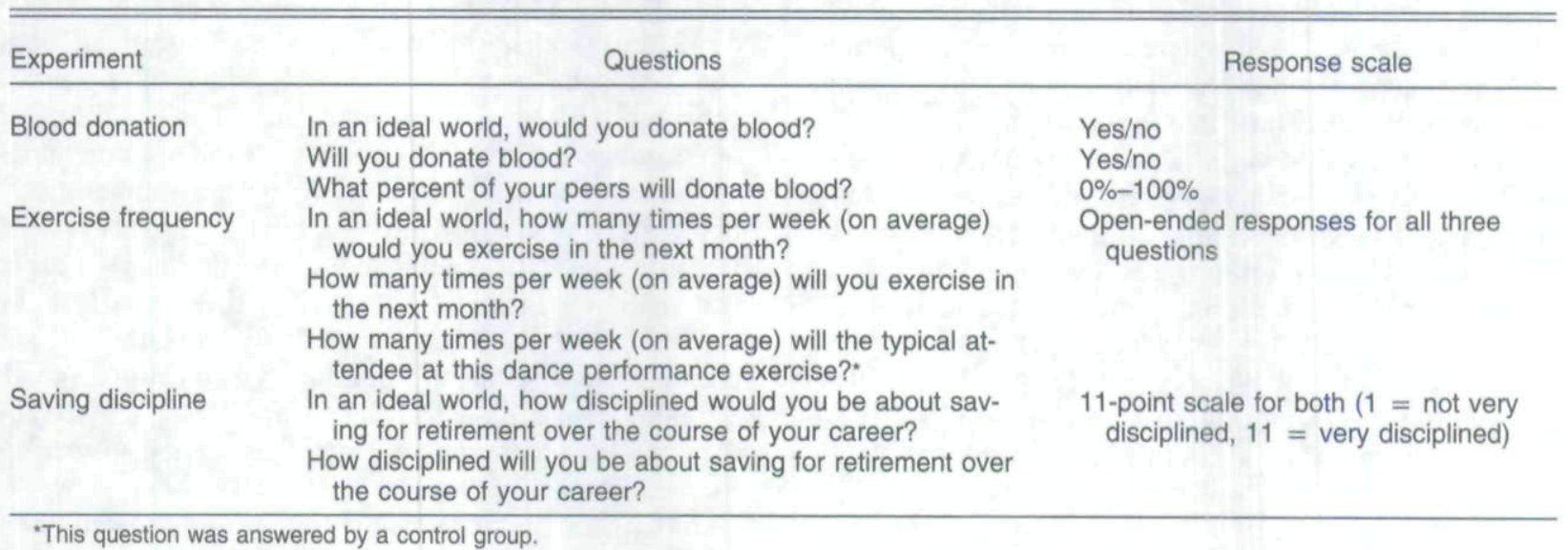

"This question was answered by a control group.

\section{Replicate Experiments}

To better understand the process underlying the data above, we ran several follow-up experiments. These replicates were identical to the experiments above, except as noted. They are reported in summary form to avoid repetition in the description of common methods.

Dual-Response Experiment. This first replicate was designed to address the possibility that participants in the ideal-first condition gave more realistic focal question estimates because answering two questions caused them to think more deeply. To examine this issue, we replicated the blood donation experiment with an additional condition in which participants estimated their blood donation likelihood $(0 \%-100 \%)$ before reading the following: "Previous research has shown that people are not always accurate when first asked for an estimate. Please take a few moments to consider again your donation likelihood." Participants then made a second donation likelihood estimate. The results ruled out a dual-response explanation. While focal estimates in the ideal-first condition $(M=40 \%)$ were reliably lower than in the standard condition $(M=71 \% ; t(25)=2.20$, $p<.04)$, estimates in the dual-response condition were identical $(M=70 \%$ for both).

Explicit Instruction Experiment. This replicate examined whether an explicit instruction to avoid idealistic predictions would produce more realistic responses. To this end, we replicated the exercise experiment and added an additional explicit instruction condition in which participants were instructed, "Please do not provide an idealistic prediction, but rather the most realistic prediction of your behavior that you can." While focal estimates in the idealfirst condition $(M=2.39)$ were lower than in the standard condition $(M=3.35)$, estimates in the explicit instruction condition were significantly higher than standard condition estimates $(M=4.37 ; t(66)=2.34, p<.05)$. This is a striking example of how difficult it can be to attenuate unrealistic optimism. That is, rather than producing realistic estimates, the explicit instruction to avoid idealistic estimates backfired, yielding higher estimates that appeared likely to be more unrealistically optimistic.

TABLE 2

BEHAVIORAL PREDICTIONS BY CONDITION-STUDY 1

\begin{tabular}{lccc}
\hline \hline & $\begin{array}{c}\text { Blood donation } \\
\text { likelihood }(\%)\end{array}$ & $\begin{array}{c}\text { Weekly exercise } \\
\text { frequency }\end{array}$ & $\begin{array}{c}\text { Savings discipline } \\
(1-11)\end{array}$ \\
\hline Focal estimates: & & & \\
Standard condition & $69.8^{\mathrm{a}, \mathrm{e}}$ & $4.72^{\mathrm{a}, \mathrm{b}}$ & $6.9^{\mathrm{d}}$ \\
Ideal-first condition & $44.1^{\mathrm{a}}$ & $3.73^{\mathrm{a}}$ & $5.3^{\mathrm{d}}$ \\
Peer estimate & $45.4^{\mathrm{e} . *}$ & $3.33^{\mathrm{b}}$ & $\mathrm{n} / \mathrm{a}$ \\
Ideal-world estimate & 69.7 & 5.03 & 7.1 \\
\hline
\end{tabular}

NOTE.-Ideal-world estimates did not differ from standard condition estimates (all three $p>4$ ).

asignificantly different, $p<.05$.

'Significantly different, $p<.05$.

'Significantly different, $p<.05$.

'Marginal difference, $p<.06$.

'No overlap in $95 \%$ confidence intervals (peer estimate continuous, self estimate binary)

"The estimated percentage of peers who would donate blood in the ideal-first condition ( $M=41.4 \%$ ) did not differ from that made by participants in the standard response condition $(M=48.5 \% ; t(74)=1.37, p>.17)$; hence a weighted average is reported. 
Decisiveness and Selective Hypothesis Testing. It is worth noting that even though we found no statistically significant difference between ideal-world and standard estimates in the studies above, there is a directional pattern in which standard estimates are slightly more realistic than ideal-world estimates. Thus, it is possible that some individuals in the standard conditions above tested both the ideal behavior hypothesis and a more realistic one. Consistent with this idea of heterogeneity in testing, there is evidence that consumers who are low in need for cognitive closure are more likely to test multiple hypotheses (Cronley et al. 2005; Kardes et al. 2004). Accordingly, we reasoned that the decisiveness factor of Webster and Kruglanski's (1994) need for cognitive closure scale (e.g., "I usually make important decisions quickly and confidently") would be a good proxy for the tendency to test multiple hypotheses in behavioral predictions. That is, those low in decisiveness should be more likely to test multiple hypotheses, including some that are realistic, when answering the standard question. Hence, we expected that individuals low in decisiveness would provide standard estimates that were farther from their ideal-world estimates than their high-decisiveness counterparts would. To test this prediction, 25 individuals estimated how often they would exercise over the next 2 weeks. Two weeks later, these same individuals estimated how many times they would exercise over the following 2 weeks in an ideal world. Two weeks later, these individuals answered the seven questions that form the decisiveness factor of the need for cognitive closure scale.

We expected that the difference between ideal-world estimates and standard condition estimates would increase as decisiveness decreased. That is, those who are most likely to consider multiple hypotheses when contemplating the standard question (i.e., those low in decisiveness) should give standard estimates that are farthest from their ideal estimates. The data supported this hypothesis. Specifically, the correlation between decisiveness and the difference measure was significant and negative $(r=-.367, p<.05)$. These data provide support for the idea that standard condition estimates stem from a process of selective hypothesis testing. Namely, greater decisiveness (which should be associated with a greater tendency toward testing a single hypothesis) was associated with less divergence between standard and ideal-world estimates.

Alternative Wording and Accuracy of Exercise Estimates. The fourth replicate had two purposes. First, we sought to determine whether an alternative wording of the ideal-world question would produce results similar to those above. Second, we wanted to determine if ideal-first participants' responses to focal questions were more accurate. All participants ( $n=56$ ) estimated how many times they would exercise over the next 2 weeks $(M=4.48)$. Two weeks later, participants reported how often they had exercised $(M=$ 3.38; $t(105)=1.90, p=.06)$. This difference is consistent with the same unrealistic optimism seen in the experiments above, except that the benchmark is actual performance, not estimates of peer performance. The day after reporting actual exercise frequency for the past 2 weeks, participants estimated how often they would exercise in the next 2 weeks. Half of the participants did so after answering a variant of the ideal-world question designed to remove all possible constraints that might impede one's ability to exercise (e.g., constraints on time, motivation, and physical ability). The question was worded as follows: "If there were no constraints on your time, motivation, and physical ability, how many times would you exercise over the next two weeks?" Because this variant removed all constraints, we expected that participants would answer it with extremely high estimates, which they did $(M=10.26)$. Nevertheless, we expected that answering this question would cause respondents to realize that the world has constraints, which would lead them to test a more realistic hypothesis when answering the focal question.

Participants in the standard condition estimated that they would exercise 4.93 times, an estimate that was significantly greater than their actual exercise frequency over the previous 2 weeks $(t(82)=2.17, p<.05)$. Thus, it seems that these participants did not use recent exercise failures to update their exercise estimates. In contrast, participants in the noconstraint condition estimated that they would exercise just 3.70 times, an estimate that was not different from actual exercise reports for the preceding 2 weeks $(t(80)=0.47$, $p>.60$ ).

From these data, we draw two conclusions. First, we have preliminary evidence that postideal responses do not just appear more realistic, but are genuinely more reflective of actual behavior. That is, if participants' self-reported exercise incidence (of three times per 2 weeks) is an accurate reflection of their actual behavior, then we can conclude that getting participants to initially consider an extremely optimistic hypothesis led to exercise estimates that were genuinely more accurate. Second, the effect of the ideal-world question (in the experiments above) does not depend on the use of the phrase "in an ideal world." We reach this conclusion because the alternative wording that emphasized the lack of constraints had a similar effect on subsequent responses to the focal question. Note, however, that the idealworld question is unique in that it produces estimates that are equal to standard condition estimates and gives rise to focal question estimates that are more realistic. As such, in all subsequent studies, we use the "in an ideal world" phrase for the initial question in the ideal-first condition.

\section{Discussion}

The data above are consistent with several of the predictions that stem from a selective hypothesis testing perspective on unrealistic optimism. First, we repeatedly observed that ideal-world behavioral estimates do not differ from standard estimates. This is consistent with the idea that participants in the standard condition are selectively testing a hypothesis of ideal behavior. Second, answering the idealworld question before answering the focal question caused participants to give more realistic estimates, which is consistent with their having tested a more realistic hypothesis. 
Third, the first two replicates ruled out a simple dualresponse explanation and also demonstrated that a direct request to provide realistic estimates was entirely ineffective in extracting realistic estimates. This suggests that correction does not follow simply from a request to correct, but rather that it requires an approach that acknowledges the underlying process and works within it. Fourth, consistent with prior research that finds that selective hypothesis testing is most prevalent among those high in need for closure, the third replicate revealed that those high in decisiveness gave standard condition estimates that were most similar to their ideal-world estimates. Finally, the last replicate showed that realistic estimates obtained when the ideal-world question was replaced by one that asked for an estimate assuming no physical, time, or motivational constraints. Though this question produced unconstrained estimates that were more extreme than the ideal-world question, it gave rise to focal question estimates that were more realistic and accurate. Thus, it is not necessary to direct attention to the idealworld hypothesis to obtain realistic estimates, but rather it is sufficient to direct attention to a hypothesis that is unrealistically optimistic.

\section{STUDY 2: DOWNSTREAM CONSEQUENCES}

An important potential consequence of being overly optimistic about one's future behavior is that such optimistic beliefs may contribute to overbuying of products that see little use (Morris and Bronson 1970). Indeed, consumers who are asked why they fail to use unused products typically cite reasons relating to a mismatch between their aspirations for product use and reality (Trocchia and Janda 2002). Not only does health and fitness equipment fit the stereotype for unused products, but evidence suggests that this category is rife with overbuying (Trocchia and Janda 2002).

The primary theoretical purpose of this study was to examine whether individuals in the ideal-first condition truly change their thinking when they answer the focal question. If they do not actually believe the more realistic estimates they report, then these estimates should not influence downstream decisions. However, if the postideal estimates are genuinely conceived, then individuals should use them when making decisions for which the estimates are relevant inputs. For example, if estimates of exercise are made more realistic (i.e., more sober) by answering the ideal-world question, then willingness to pay for exercise equipment should fall.

Methodologically, this study follows the exercise experiments above, with one change. After answering the focal exercise estimation question, participants in both conditions reported their willingness to pay for a new treadmill. We expect that participants who estimate their exercise frequency after answering the ideal-world question will report lower willingness to pay for a treadmill than participants who estimate exercise frequency in the standard condition.

\section{Method}

Participants were 118 undergraduate students, each of whom was randomly assigned to either the standard or idealfirst exercise condition from study 1 . After estimating their exercise, they reported willingness to pay for a treadmill.

\section{Results}

To begin, we verified that exercise estimates in the standard condition $(M=3.16)$ were higher than focal question estimates in the ideal-first condition $(M=2.47 ; t(116)=$ $2.20, p<.05$ ). Thus, the effect that the ideal-world question has on answers to the focal question (i.e., making them more realistic) was replicated here. Of greater interest, participants in the ideal-first condition reported a lower willingness to pay for the treadmill $(M=\$ 480)$ than participants in the standard condition $(M=\$ 610 ; t(116)=2.00, p<.05)$. Thus, it appears that exercise estimates were not just arbitrary numbers that our participants produced, but they are meaningful inputs to downstream decisions.

\section{Discussion}

A remaining question is whether answering the idealworld question causes everyone to adjust their estimates toward the more pessimistic end of the response set. If so, then those who would have given realistic estimates without the intervention might give overly pessimistic estimates. Note, however, that the selective hypothesis testing account predicts that the ideal-world question will cause only those consumers who would default to an idealistic hypothesis to adjust their behavioral estimates. In other words, estimates of those who would default to a realistic hypothesis in the standard condition should not be influenced by the idealworld question. As such, we expect that answering the idealworld question will not uniformly influence all consumers. One goal of the next study is to explore this issue.

\section{STUDY 3: PREDICTING PRODUCT USAGE AND WILLINGNESS TO PAY}

This study has two goals. First, it examines whether the effects above extend to predictions of product usage (i.e., how many songs participants predict they would load onto a new iPod). If so, then this work might have much to say about helping consumers avoid overbuying in a variety of domains. We examine the issue of overbuying by collecting willingness to pay for a new 60-gigabyte (GB) iPod after iPod usage estimates are collected.

Our second goal was to further test our claim that selective hypothesis testing contributes to unrealistically optimistic predictions of future behavior. Following previous research that has found that experts are less likely (relative to novices) to exhibit behavior that is consistent with selective hypothesis testing (Posavac et al. 2005), we examine product expertise as a potential moderator. We use iPod ownership as a proxy for expertise because owners should be more knowl- 
edgeable about their likely capacity utilization of an iPod. This knowledge comes with a better understanding of the constraints associated with iPod utilization (e.g., the time and effort required to load songs onto the iPod). We expect that experts will be less likely to adopt ideal capacity utilization as their tentative hypothesis when estimating their iPod utilization. As such, standard condition estimates by experts should be more realistic (i.e., lower) than ideal-world estimates by experts. Additionally, focal question estimates by experts should not differ across conditions. In contrast, novices should display the same pattern we have seen in every experiment so far. To the extent that iPod valuation is contingent on capacity utilization, novices should report lower willingness to pay for an iPod in the ideal-first condition than in the standard condition, whereas experts should give similar estimates in both. Such a pattern would reveal whether answering the ideal-world question causes all consumers to reduce their willingness to pay for a product or whether it works only where appropriate.

\section{Method}

This study employed a 2 (iPod expertise: own vs. do not own) $\times 2$ (request condition: ideal-first vs. standard) between-subjects design, with song storage and willingness to pay for a new iPod as dependent variables. Participants were 227 undergraduate and postgraduate students. Nine surveys were completed incorrectly, leaving 218 participants on which all analyses were performed. Each participant saw a picture of an iPod and read the following text:

At just over half an inch thick, the iPod fits comfortably in the palm of your hand and slips easily into your pocket-and your life. Merely 5.6 ounces, it weighs less than two compact disks, and even many cell phones. And yet the iPod gives you a huge 60GB hard drive- big enough to hold 15,000 songs.

Participants in the ideal-first condition answered the two following questions, and those in the standard condition answered just the second question.

In an ideal world, how many songs would be loaded on your iPod at any time?

How many songs would be loaded on your iPod at any time?

Participants in both conditions were then told to assume that they did not own an iPod and to report their willingness to pay for a $60 \mathrm{~GB}$ model in response to the following question: "How much would you be willing to pay for this iPod?" Open-ended responses were recorded in dollars. Finally, to measure expertise, participants were asked if they currently owned an iPod.

\section{Results}

For nonowners $(n=67)$, song storage estimates were higher in the standard request condition $(M=4,854)$ than in the ideal-first condition $(M=1,993 ; t(63)=2.76, p<$ $.01)$. Furthermore, consistent with the emerging pattern from the studies to date, ideal-world estimates in the ideal-first condition $(M=4,680)$ did not differ from standard condition estimates $(p>9)$. In contrast, for iPod owners ( $n=151)$, ideal-world estimates in the ideal-first condition ( $M=7,676)$ were dramatically higher than standard condition estimates $(M=4,184 ; t(151)=4.00, p<.01)$. Additionally, focal estimates by iPod owners in the ideal-first condition $(M=3,469)$ did not differ from standard condition estimates $(t(151)=1.04, p=.30)$. Both of these findings suggest that the song storage estimates by iPod owners were less affected by selective hypothesis testing of ideal usage. That is, because iPod owners are more aware of the constraints associated with iPod utilization (e.g., costs of loading songs onto the iPod), they were less likely to default to an ideal hypothesis in the standard condition.

Next we examined the willingness to pay estimates. Figure 1 plots willingness to pay by condition. A two-way ANOVA of willingness to pay yielded a main effect of ownership $(F(1,214)=22.27, p<.005)$, a marginal main effect of request condition $(F(1,120)=3.33, p<.07)$, and an ownership by request condition interaction $(F(1$, $214)=4.40, p<.04)$. We conducted planned contrasts to expose the nature of the interaction. As expected, nonowners in the ideal-first condition reported a lower willingness to pay $(M=\$ 129)$ than nonowners in the standard condition $(M=\$ 188 ; F(1,214)=5.42, p<.03)$. For iPod owners, willingness to pay in the ideal-first condition $(M=\$ 231)$ did not differ from that in the standard condition $(M=$ $\$ 227 ; p>0.9$ ). As with iPod usage, the higher willingness to pay by iPod owners probably reflects greater preference for the iPod in general. It is worth noting that this difference in average willingness to pay is inconsistent with the notion that the null effect for iPod owners was due to a floor effect

\section{FIGURE 1}

IPOD WILLINGNESS TO PAY FOR OWNERS AND NONOWNERS BY QUESTION CONDITION-STUDY 3

iPod \$ WTP

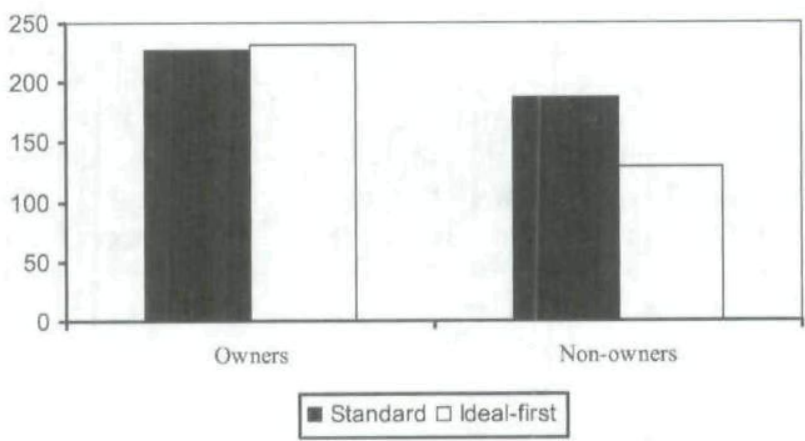


(i.e., that they were unwilling to pay anything because they already owned one).

\section{Discussion}

In this study we explored a situation in which the focal question required a prediction of how fully one would use the capacity of a specific product (i.e., iPod capacity utilization). Results indicated that whereas ideal-world and standard estimates were similar for novices (consistent with prior studies), they were dramatically different for experts, with the ideal-world estimate being considerably higher. Furthermore, prior consideration of ideal usage did not influence focal question usage estimates for experts but had the expected effect on estimates of novices. Both findings are consistent with the idea that experts are less likely to default to adopting an ideal usage hypothesis and thus further support our selective hypothesis testing case.

These findings also suggest that consumers who lack experience with a product are most at risk of making idealized future usage predictions and thus are most at risk of making ill-advised purchases. Though troubling, these data also offer hope. Namely, consumers who initially consider their ideal product usage subsequently think more realistically about their likely usage. For example, if the novice data above held in an Apple store, then it is possible that a consumer who used the ideal-world question on herself would realize that trading down from a $60 \mathrm{~GB}$ to a $20 \mathrm{~GB}$ iPod would be optimal.

\section{STUDY 4: THE PLANNING FALLACY}

People routinely underestimate how long it will take them to finish tasks. This "planning fallacy" has been documented in everything from long-term construction projects to much briefer laboratory tasks (Buehler, Griffin, and Ross 1994; Kahneman and Tversky 1979). The tendency to underestimate how long it will take to complete a task has potential consequences both for consumers themselves and for those who rely on their estimates. Examples of the former include lost savings from failing to return rebate documentation on time, choosing a high-interest/high-benefit credit card on the basis of the optimistic expectation that bills will be paid on time, and the cost associated with adjusting one's activities to address overcommitment. With respect to consequences for others, there are numerous examples in which the failure to complete a particular task on schedule causes delays in related projects that can result in cascading inefficiencies in resource allocation.

This study consists of two related experiments. The primary goal of the first experiment (henceforth described as the "planning accuracy" experiment) was to ascertain whether answering the ideal-world question would cause participants to render longer and more accurate estimates of task completion. A second goal was to investigate whether predicting longer completion times undermines actual task completion. That is, would realistic estimates give rise to longer actual completion times, or would such estimates have no effect on task completion? The goal of the second experiment (henceforth described as the "planning thoughts" experiment) was to examine whether participants in the ideal-first condition thought more realistically when answering the focal question. To examine this question, we collected retrospective thought listings. Participants in both experiments estimated when they would complete a task (watching a digital video disc [DVD] for a class project or completing a hypothetical leisure time report). In both cases we expected that participants in the ideal-first condition would provide longer completion time estimates when answering the focal question.

\section{Planning Accuracy Experiment}

Method. Participants in this experiment were 95 executive Master of Business Administration students who participated as part of an in-class exercise. They were given a DVD that contained a 1-hour focus group discussion, which they had to watch as a first step to completing a group project that was due in 2 weeks. In the same class in which the DVDs were distributed, participants predicted when they would watch the DVD. Half of the participants did so after estimating when they would ideally watch the DVD: "In an ideal world, when would you finish watching the DVD?" The other half simply estimated when they would watch the DVD. Actual task completion times were collected 2 weeks later, with all participants answering the following question: "When did you watch the DVD?" Participants reported dates that were translated into the number of days from the day on which the DVD was distributed. Thus, the minimum possible value was 0 (watched it on the same day it was received) and the maximum value was less than 14 (watched it the day the assignment was due).

Results. Average completion time estimates and average actual completion times are presented in column 1 of table 3. As in earlier studies, there was no difference between standard and ideal-world estimates. However, focal question estimates in the ideal-first condition were significantly longer than those of participants in the standard condition. Of greater importance, the average completion time estimates for those in the ideal-first condition ( $M=4.43$ days) did not differ from how long participants actually took to watch the DVD ( $M=4.45$ days). In other words, participants in the ideal-first condition gave estimates that were, on average, objectively accurate.

Next, we considered whether participants in the ideal-first condition (who gave longer predictions of completion time) took longer to watch the DVD than participants in the standard condition. We observed no evidence of this: the actual time to watch the DVD did not differ between the idealfirst and standard conditions $(t(93)=1.02, p>.30)$. This suggests that providing more realistic completion time estimates did not undermine actual performance of ideal-first participants. 
TABLE 3

ESTIMATED TASK COMPLETION TIMES-STUDY 4

\begin{tabular}{|c|c|c|}
\hline & $\begin{array}{l}\text { Planning accuracy } \\
\text { study (watch DVD) } \\
\text { (1) }\end{array}$ & $\begin{array}{c}\text { Planning thoughts } \\
\text { study (complete report) } \\
\text { (2) }\end{array}$ \\
\hline \multicolumn{3}{|c|}{ Completion time estimates: } \\
\hline Standard condition & 3.04 days $^{\mathrm{a}, \mathrm{d}}$ & 1.5 days $^{\mathrm{b}, c}$ \\
\hline Ideal-first condition & 4.43 days $^{\mathrm{a}, \theta}$ & 2.5 days $^{b}$ \\
\hline Actual completion time & 4.45 days $^{\circ}$ & NA \\
\hline Ideal-world estimate & 2.74 days $^{d}$ & 1.0 day $^{c}$ \\
\hline
\end{tabular}

aSignificantly different, $p<.05$.

'Significantly different, $p<.05$.

"Marginal difference, $p<.09$.

No difference, $p>.4$.

'No difference, $p>9$.

\section{Planning Thoughts Experiment}

Method. Participants were 56 students who were told that the purpose of the experiment was to test materials for research in which participants would have to write a report on how they spent their leisure time for a specific day. They were informed that they would not actually have to write the report, but that they should complete all materials and think about the report-writing task exactly as if they had to do so. The materials stated that the leisure report could pertain to any day from the upcoming week. Instructions also stated that since memory fades quickly, the report needed to be written within 5 days of the day they chose to chronicle.

Participants were randomly assigned to either the standard or ideal-first condition and made completion time predictions as follows. Those in the ideal-first condition answered both of the questions below, whereas those in the standard condition answered just the second question.

In an ideal world, when would you expect to complete the report?

When do you expect to complete the report?

After this, participants were asked to describe the major thoughts they had as they answered the focal question.

Results. Completion time estimates in this experiment were derived by subtracting the date they predicted they would write the report from the earliest possible day they could write the report. Average completion times appear in column 2 of table 3 . These data reveal the same pattern as we have seen routinely in our studies (i.e., no difference between standard and ideal-world estimates and postideal estimates that are more realistic than standard condition estimates). Next, we examined the thought listing data.

We reasoned that the primary reasons for completing a class project more slowly than expected would pertain to procrastination and weak motivation (i.e., putting it off until the last minute). Thus, if participants in the ideal-first condition were thinking more realistically, thoughts relating to procrastination and/or weak motivation should be more accessible. Hence, thought listings were coded by two independent coders for mention of either procrastination or weak motivation as reasons contributing to the report completion estimate. Agreement between the raters was high $(r=.88)$, and disagreements were resolved through discussion.

Analysis revealed that a greater percentage of participants in the ideal-first condition mentioned procrastination or weak motivation as an issue involved with project completion $(M=33 \%)$ than individuals in the standard request condition $\left(M=7 \% ; \chi^{2}=5.54, p<.03\right)$. That is, those in the ideal-first condition engaged in more realistic thinking when formulating their completion time estimates than participants in the standard condition. This result is interesting in its own right since a significant body of research suggests that individuals are loath to admit volitional responsibility for negative outcomes. For example, research on postdecision counterfactuals suggests that individuals would rather construct external reasons for poor performance than assign any personal liability (Gilbert et al. 2004).

To explore the role of these thoughts more fully, we performed a mediation analysis following Baron and Kenny (1986). Figure 2 displays the regression coefficients for the key relationships in this analysis. There was a significant relationship between question condition and reported procrastination thoughts $(a)$, question condition and participants' estimated report completion time $(b)$, and procrastination thoughts and estimated report completion time $(c)$. The relationship between question condition and estimated report completion time was not significant when controlling for procrastination thoughts $(d)$, and the reduction in the beta versus the model without the mediator is significant by a Sobel test $(z=1.95, p=.05)$. Thus, there is evidence that the effect of condition on completion time estimates was mediated by realistic thoughts.

\section{Discussion}

The data from these two experiments suggest that initially estimating ideal task completion time results in subsequent completion estimates that are more accurate and that the 
FIGURE 2

PROCRASTINATION-RELATED THOUGHTS MEDIATE ATTENUATION OF THE PLANNING FALLACY-STUDY 4

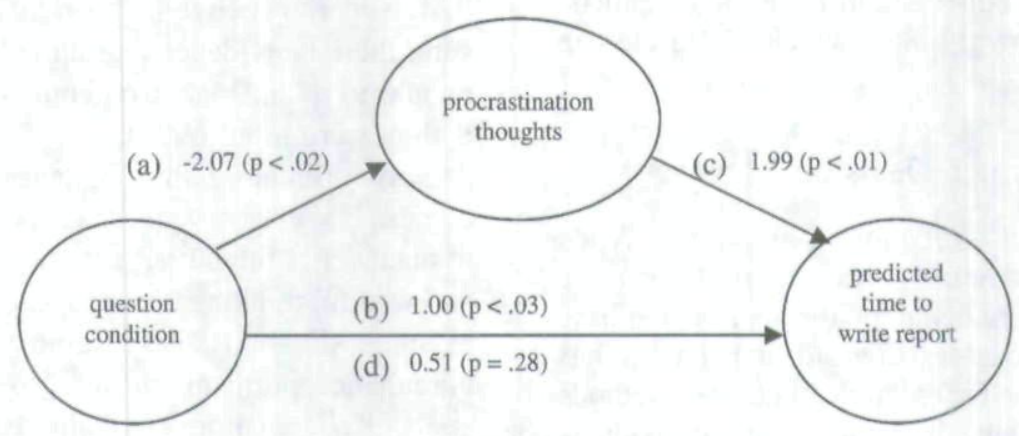

process of generating those estimates is governed by more realistic thinking, not just a simple adjustment process. Put differently, both the estimates themselves and the thought listings strongly suggested that answering the ideal-world question caused participants to think more realistically when answering the focal question.

\section{GENERAL DISCUSSION}

Across a multitude of studies and replicates we consistently found that predictions of future behavior were nearly identical to those made by individuals who were explicitly instructed to give estimates assuming conditions would be ideal. We also found that individuals who first answered the ideal-world question subsequently provided more realistic estimates of future behavior (i.e., gave estimates that did not differ from peer estimates or from actual behavior). Though these individuals reported more realistic thoughts, we found no evidence that this realistic thinking undermined actual performance. These findings have important implications for theories of unrealistic optimism and consumer decision making.

\section{Theoretical Contributions}

Our data contribute to the unrealistic optimism literature in several ways. First, the totality of our data suggests that selective hypothesis testing contributes to unrealistic optimism. The evidence to support this is as follows. As noted above, standard estimates were routinely as high as idealworld estimates. An explanation for this similarity is that standard estimates are constructed around a hypothesis of ideal behavior. Moreover, if unrealistic optimism derives from selective testing of an ideal hypothesis, then it should be possible to ameliorate it by getting individuals to move away from their ideal hypothesis. We believe that this is precisely the effect that answering the ideal-world question had on our participants. Specifically, participants who initially answered the ideal-world question subsequently predicted future behaviors that did not differ from estimates of peer behavior and were more in line with actual future behavior. Additionally, consistent with previous work (Kardes et al. 2004), we found that greater decisiveness was associated with smaller differences in standard and ideal-world estimates. Finally, the expertise moderation results in study 3 are consistent with prior work that finds that experts are less likely to engage in selective hypothesis testing (Posavac et al. 2005).

Another contribution, albeit subtle, stems from the result that standard estimates were often indistinguishable from ideal estimates. This by itself suggests a more nuanced view of unrealistic optimism. Specifically, while it is a common finding that individuals are too optimistic in predictions of their future behavior, the current work reveals that behavioral predictions are not simply too high, but rather often line up with perceptions of ideal behavior.

Third, we note that recent research in the areas of leaderdriven primacy and selective hypothesis testing (Bond et al. 2007; Carlson, Meloy, and Russo 2006; Posavac et al. 2004) has made great strides in identifying processes whereby initial leanings or hypotheses become reinforced over time, leading ultimately to stronger preferences or views than originally existed. The current research adds to this literature on selective hypothesis testing by extending our understanding of the types of hypotheses individuals test. That is, when estimating future behavior, they appear to test the hypothesis that they will act in an idealistic fashion.

A fourth theoretical contribution of this work is that our findings lend support to biased retrieval accounts of unrealistic optimism. These accounts contrast with biased encoding views that contend that many forms of self-favoritism result from the selective integration of self-favoring information into memory (Baumeister and Cairns 1992). The current data are difficult to reconcile with biased encoding because the ideal-world question did not give participants information about themselves that they did not already possess.

A final contribution to the literature on unrealistic optimism comes from the planning accuracy study (see study 4). Recall that compared to participants in the standard condition, participants who predicted their completion times after answering the ideal-world question gave longer and more accurate completion estimates, but they did not take any longer to actually complete the task. This suggests that 
the ideal-world approach for getting people to adopt a more realistic hypothesis does not necessarily undermine performance. This result is noteworthy since it contributes to the ongoing debate about the benefits and costs of unrealistic optimism (Taylor and Brown 1988), which we discuss in greater detail below.

\section{Implications for Consumer Decision Making}

Debiasing Estimates of Consumer Behavior. When viewed as a technique for helping consumers think more realistically about their future behavior, the approach of having consumers initially consider their ideal behavior has substantial appeal. Consider, for example, that most methods for inducing realistic estimates implicitly direct individuals to move their estimates in a particular direction (e.g., Hoch 1985; Lord, Lepper, and Preston 1984). These consider the opposite approaches require that the administrator know the direction and magnitude of the bias in advance. Moreover, they implore adjustment by all members of the population. As such, they risk creating an opposite bias in those who would not otherwise be unrealistically optimistic.

As a pure debiasing technique, the ideal-world approach does not share these shortcomings. Since each individual's notion of ideal is different, it is not necessary to know the size or even the direction of a bias for the technique to work. Moreover, it has the potential to work on both sides of a problem at once. Consider two college-age men, one who wants to gain muscle weight and one who wants to lose weight. When asked "In an ideal world, how much would you weigh in 2 months?" they will produce different answers, with one producing an answer that is likely to be higher and the other producing an answer that is likely to be lower than their current weights, respectively. When subsequently asked "How much will you weigh in 2 months?" they should each give answers that are more realistic than they would otherwise, presumably closer to their current weight. Thus, even without knowing in which direction a specific person is likely to bias his forecast, it should be possible to elicit more realistic estimates by having him first estimate his ideal.

Consequences for Decision Quality. While the primary purpose of our research was to explore a selective accessibility explanation for unrealistic optimism, our findings may have implications for the quality of consumer decisions. In particular, it is possible that getting consumers to think more realistically will influence the quality of their decisions.

Research relevant to this question can be found in a number of literatures. First, a large body of research in psychology argues that optimism actually has positive consequences for mental health and/or individual welfare (Peterson 1988; Scheier et al. 1989; Weisse 1992). Most of this research is based on the finding that high or exaggerated expectations often have a self-fulfilling property, with illusory optimism strengthening motivation and aspirations, leading ultimately to superior performance (Taylor and
Brown 1988). The evidence for this is particularly strong in health domains. For example, Scheier et al. (1989) observed that optimistic heart bypass patients made substantially superior recoveries than their less optimistic peers. In sum, there is evidence that unrealistic optimism can enable people to attain higher outcomes than they would achieve if they were more realistic.

Notwithstanding this evidence for positive health outcomes, however, a significant body of research, primarily in managerial literatures, argues that optimistic expectancies and wishful thinking can also lead to negative consequences in some situations. For example, it has been argued that unrealistic optimism can lead to the pursuit of unrealistic goals (Kahneman and Lovallo 1993), distraction from the formation of implementation plans (Oettingen 1996), poor decision making (Baumeister 1989), poor negotiation (Neale and Bazerman 1985), and the excessive consumption of vice products (Khan and Dhar 2007).

The possibility of unrealistic optimism having both positive and negative consequences leaves the welfare picture somewhat muddied. That is, while consumers who think more realistically may make better decisions in the present, realistic thinking may also undermine actual future behavior. Hence, an important area for future research is to understand when and how future behavior is influenced by expectations of future behavior.

One way to frame the issue is in terms of goals. Specifically, prior research suggests that consumers who construct behavioral intentions sometimes treat these intentions as goals (Alexander, Lynch, and Wang 2008; Levav and Fitzsimons 2006). Since goals are motivating, expectations of future behavior that are treated as goals should be motivating. If so, then a key issue in knowing whether the induction of realistic expectations will undermine performance is whether consumers will treat their expectations as goals. Since abstract expectations are less goal-like (and less motivating) than concrete ones (Levav and Fitzsimons 2007), the crux of this issue might reside in the extent to which expectations are represented abstractly or concretely. For example, one possibility is that postideal estimates may be built from more concrete representations of the future and thus might actually be more goal-like than the more abstract standard estimates. Thus it is far from clear what the net impact on motivation will be since even though postideal estimates are less lofty than standard estimates, which may make them less motivating, they may also be more goallike, which may increase motivation.

What is clear is that it is not possible to provide a definitive answer as to how increased realism might affect consumer welfare without a better understanding of how behavioral expectancies influence actual behavior. As such, future research might focus on identifying the conditions under which performance is degraded by more realistic estimates and the conditions under which it is not. 


\section{Ruling Out Demand}

Experimental demand (Shimp, Hyatt, and Snyder 1991) is a concern whenever participants might be able to guess the research hypothesis. Since the similarity between idealworld and standard estimates is a between-condition result, the data for which demand is most a concern are the postideal estimates. That said, it is hard to make the case for a parsimonious demand account of the postideal data that explains all of our studies.

First, it is not clear why participants would conclude that they should contrast from their ideal-world estimates. Indeed, another possibility is that ideal-first participants would anchor on their ideal-world estimates, which would eventually yield postideal estimates that were too optimistic. Second, even if demand does lead participants to adjust in the hypothesized direction, ideal-first participants did more than simply adjust from their ideal-world estimates. They gave focal question responses that $(a)$ were indistinguishable both from their own peer estimates (study 1, blood donation) and from peer estimates of a control group (study 1, exercise); (b) were not different from actual recent behavior (study 1, third replicate); and $(c)$ did not differ from actual future behavior (study 4, accuracy study). Third, in a study in which participants did not need to infer the wishes of the experimenters (i.e., in which participants were told to avoid ideal estimates and to provide realistic ones), participants did not give realistic estimates. If experimental demand were at work in our studies, we should have seen very realistic estimates in this replicate. Fourth, demand accounts do not generally extend beyond the measure of interest. However, thought listings from study 4 and the downstream willingness to pay estimates in studies 2 and 3 indicate that the effect of answering an ideal-world question extended beyond the postideal focal question responses. Finally, demand is inconsistent with the expertise results in study 3 . In particular, it is highly unlikely that participants could intuit and then deliver (in a between-subjects design) the pattern of results that we observed.

\section{Conclusion}

Consistent with theories of self-determination (e.g., Deci and Ryan 1985), we find that the key to helping consumers make realistic predictions is helping them to do so of their own accord. Namely, our studies reveal that realistic predictions do not follow from requests to be realistic, but rather from an approach that works within the underlying process of selective hypothesis testing. In other words, the key to more realistic predictions of future behavior lies not in exhorting consumers to ignore the ideal but in getting them to acknowledge it. Those who did so of their own accord abandoned their idealistic expectations and embraced realistic ones.

\section{REFERENCES}

Alexander, David, John G. Lynch Jr., and Qing Wang (2008), “As Time Goes By: Warm Intentions and Cold Feet for ReallyNew vs. Incrementally-New Products," Journal of Marketing Research, 45 (3), 307-19.

Armor, David A. and Shelley E. Taylor (2002), "When Predictions Fail: The Dilemma of Unrealistic Optimism," in Heuristics and Biases: The Psychology of Intuitive Judgment, ed. Thomas D. Gilovich, Dale Griffin, and Daniel Kahneman, Cambridge: Cambridge University Press, 334-47.

Baron, Reuben M. and David A. Kenny (1986), "The ModeratorMediator Variable Distinction in Social Psychological Research: Conceptual, Strategic, and Statistical Considerations," Journal of Personality and Social Psychology, 51 (1), 1173-82.

Baumeister, Roy F. (1989), "The Optimal Margin of Illusion," Journal of Social and Clinical Psychology, 8 (2), 176-89.

Baumeister, Roy F. and Kenneth J. Cairns (1992), "Repression and Self-Presentation: When Audiences Interfere with SelfDeceptive Strategies," Journal of Personality and Social Psychology, 62 (3), 851-62.

Bond, Samuel D., Kurt A. Carlson, Meg G. Meloy, J. Edward Russo, and Robin J. Tanner (2007), "Precommitment Bias in the Evaluation of a Single Option," Organizational Behavior and Human Decision Processes, 102 (2), 240-54.

Buehler, Roger, Dale Griffin, and Michael Ross (1994), "Exploring the 'Planning Fallacy': Why People Underestimate Their Task Completion Times," Journal of Personality and Social Psychology, 67 (3), 366-81.

Burger, Jerry M. and Linda Burns (1988), "The Illusion of Unique Invulnerability and the Use of Effective Contraception," Personality and Social Psychology Bulletin, 14 (2), 264-70.

Carlson, Kurt A., Margaret G. Meloy, and J. Edward Russo (2006), "Leader-Driven Primacy: Using Attribute Order to Affect Consumer Choice," Journal of Consumer Research, 32 (March), 513-18.

Cronley, Maria L., Steven S. Posavac, Tracy Meyer, Frank R. Kardes, and James J. Kellaris (2005), "A Selective Hypothesis Testing Perspective on Price-Quality Inference and InferenceBased Choice," Journal of Consumer Psychology, 15 (2), 159-69.

Deci, Edward L. and Richard R. Ryan (1985), Intrinsic Motivation and Self-Determination in Human Behavior, New York: Plenum.

Deighton, John (1984), "The Interaction of Advertising and Evidence," Journal of Consumer Research, 11 (December), 763-70.

Gettys, Charles G. and Stanley D. Fisher (1979), "Hypothesis Plausibility and Hypothesis Generation," Organizational Behavior and Human Performance, 25 (1), 93-110.

Gilbert, Daniel T., Carey K. Morewedge, Jane L. Risen, and Timothy D. Wilson (2004), "Looking Forward to Looking Backward: The Misprediction of Regret," Psychological Science, 15 (5), 346-50.

Hoch, Stephen J. (1985), "Counterfactual Reasoning and Accuracy in Predicting Personal Events," Journal of Experimental Social Psychology: Learning, Memory, and Cognition, 11 (4), 719-31.

Hoch, Stephen J. and John Deighton (1989), "Managing What Consumers Learn from Experience," Journal of Marketing, 53 (2), 1-20.

Hoorens, Vera (1995), "Self-Favoring Biases, Self-Presentation 
and the Self-Other Asymmetry in Social Comparison," Journal of Personality, 63 (4), 793-817.

Kahneman, Daniel and Dan Lovallo (1993), "Timid Choices and Bold Forecasts: A Cognitive Perspective on Risk Taking," Management Science, 39 (1), 17-31.

Kahneman, Daniel and Amos Tversky (1979), "Intuitive Prediction: Biases and Corrective Procedures," Management Science, 12 (March), 313-27.

- (1982), "The Simulation Heuristic," in Judgment under Uncertainty: Heuristics and Biases, ed. Daniel Kahneman, Paul Slovic, and Amos Tversky, Cambridge: Cambridge University Press, 201-10.

Kardes, Frank R., Maria L. Cronley, James J. Kellaris, and Steven S. Posavac (2004), "The Role of Selective Information Processing in Price-Quality Inference," Journal of Consumer Research, 31 (September), 368-74.

Khan, Uzma and Ravi Dhar (2007), "Where There Is a Way, Is There a Will? The Effect of Future Choices on Self Control," Journal of Experimental Psychology: General, 136 (2), 277-88.

Kruger, Justin and Thomas Gilovich (2004), "Actions, Intentions, and Self-Assessment: The Road to Self-Enhancement Is Paved with Good Intentions," Personality and Social Psychology Bulletin, 30 (3), 328-39.

Levav, Jonathan and Gavan J. Fitzsimons (2006), "When Questions Change Behavior: The Role of Ease of Representation," Psychological Science, 17 (3), 207-13.

Lord, Charles G., Mark R. Lepper, and Elizabeth Preston (1984), "Considering the Opposite: A Corrective Strategy for Social Judgment," Journal of Personality and Social Psychology, 47 (6), 1231-43.

McKenna, Frank P. (1993), "It Won’t Happen to Me: Unrealistic Optimism or Illusion of Control?" British Journal of Psy. chology, 84 (1), 39-50.

Morris, Ruby T. and Claire S. Bronson (1970), "The Potential Loss in Money Income to the American People in Haphazard Purchasing," Journal of Consumer Affairs, 4 (2), 103-12.

Neale, Margaret A. and Max H. Bazerman (1985), "The Effects of Framing and Negotiator Overconfidence on Bargaining Behaviors and Outcomes," Academy of Management Journal, 28 (1), 34-49.

Newby-Clark, Ian R. (2005), "Plans and Predictions for Exercise Frequency Change," Basic and Applied Social Psychology, 27 (2), 97-106.

Oettingen, Gabriele (1996), "Positive Fantasy and Motivation," in The Psychology of Action: Linking Cognition and Motivation to Behavior, ed. P. M. Gollwitzer and J. A. Bargh, New York: Guilford, 236-59.

Perloff, Linda S. and Barbara K. Fetzer (1986), "Self-Other Judgments and Perceived Vulnerability to Victimization," Journal of Personality and Social Psychology, 50 (3), 502-10.

Peterson, Christopher (1988), "Explanatory Style as a Risk Factor for Illness," Cognitive Therapy and Research, 12 (2), 119-32.

Posavac, Steven S., Frank R. Kardes, David M. Sanbonmatsu, and Gavan J. Fitzsimons (2005), "Blissful Insularity: When Brands Are Judged in Isolation from Competitors," Marketing Letters, 16 (2), 87-97.
Posavac, Steven S., David M. Sanbonmatsu, Frank R. Kardes, and Gavan J. Fitzsimons (2004), "The Brand Positivity Effect: When Evaluation Confers Preference," Journal of Consumer Research, 31 (3), 643-51.

Sanbonmatsu, David M., Steven S. Posavac, Frank R. Kardes, and Susan P. Mantel (1998), "Selective Hypothesis Testing," Psychonomic Bulletin and Review, 5 (June), 197-220.

Scheier, Michael F., Karen A. Matthews, Jane F. Owens, George J. Magovern, Craig R. Lefebvre, R. Anne Abbott, and Charles S. Carver (1989), "Dispositional Optimism and Recovery from Coronary Artery Bypass Surgery: The Beneficial Effects on Physical and Psychological Well-Being," Journal of Personality and Social Psychology, 57 (6), 1024-40.

Shimp, Terence A., Eva M. Hyatt, and David J. Snyder (1991), "A Critical Appraisal of Demand Artifacts in Consumer Research," Journal of Consumer Research, 18 (December), 273-83.

Smith, Steven M., Leandre R. Fabrigar, Deborah M. Powell, and Marie-Jöelle Estrada (2007), "The Role of Information-Processing Capacity and Goals in Attitude-Congruent Selective Exposure Effects," Personality and Social Psychology Bulletin, 33 (7), 948-60.

Soll, Jack B., Richard P. Larrick, and Xiumei Zhu (2006), "The Wisdom of the Crowd in the Mind," paper presented at Behavioral Decision Research in Management, Santa Monica, CA.

Taylor, Shelley E. and David A. Armor (1996), "Positive Illusions and Coping with Adversity," Journal of Personality, 64 (4), 873-98.

Taylor, Shelley E. and Jonathon D. Brown (1988), "Illusion of Well-Being: A Social Psychological Perspective on Mental Health," Psychological Bulletin, 103 (2), 193-210.

Trocchia, Philip J. and Swinder Janda (2002), "An Investigation of Product Purchase and Subsequent Non-consumption," Journal of Consumer Marketing, 19 (3), 188-204.

Wason, Peter C. (1971), "Natural and Contrived Experience in a Reasoning Problem," Quarterly Journal of Experimental Psychology, 23 (1), 63-71.

Webster, D. M. and Arie W. Kruglanski (1994), "Individual Differences in Need for Cognitive Closure," Journal of Personality and Social Psychology, 67 (6), 1049-62.

Weinstein, Neal D. (1980), "Unrealistic Optimism about Future Life Events," Journal of Personality and Social Psychology, 39 (5), 806-20.

- (1987), "Unrealistic Optimism about Susceptibility to Health Problems: Conclusions from a Community-wide Sample," Journal of Behavioral Medicine, 10 (5), 481-500.

Weisse, Carole S. (1992), "Depression and Immunocompetence: A Review of the Literature," Psychological Bulletin, 111 (3), 475-89.

Williams, Elanor F. and Thomas Gilovich (2006), "The Best I Can Be: Best Performances Are Representative Performances for the Self," paper presented at the Midwestern Psychological Association annual meeting, Chicago.

Wills, Thomas A. (1981), "Downward Comparison Principles in Social Psychology," Psychological Bulletin, 90 (2), 245-71. 
Copyright of Journal of Consumer Research is the property of Journal of Consumer Research. Inc. and its content may not be copied or emailed to multiple sites or posted to a listserv without the copyright holder's express written permission. However, users may print, download, or email articles for individual use. 